\title{
Catecholamine in Gongestive Heart Failure (Report I)
}

\author{
Tatsuya Tomomatsu, M.D., Yasuyuki U $\mathrm{UBa}_{\text {, }}$ M.D., \\ Tetsuo Matsumoto, M.D., Teishi Iкомa, M.D., \\ and Yoshihiko Kondo, M.D.
}

\begin{abstract}
In order to determine the role of the catecholamines in cardiac failure, we considered the urinary excretion of catecholamines on cardiac patients suffering from acquired valvular disease.

Among those who were admitted to the ward of Kobe Medical College Hospital, 178 patients with the congestive cardiac failure were chosen. These patients have been divided into four groups according to the criteria of New York Heart Association. Values of both urinary adrenaline and noradrenaline were progressively increased from grade I to grade III of congestive heart failure and reduced in grade IV but not to the normal range.

Urinary excretion of both adrenaline and noradrenaline had increased when the conditions were improved from grade IV to grade III and reduced with further restoration from grade III to grade II. When the conditions took a turn for the worse, urinary excretion of active catechols varied inversely.

The measurements of urinary active catechols were made on every 5 or 6 hour urine during the course of the rapid digitalization and compared with some clinical features.

Some comments were tried on the significance of urinary excretion of active catechols.
\end{abstract}

SIGNIFICANCE of catecholamines in cardiac disease is one of the interesting and challenging problems. In 1954, Raab ${ }^{1)}$ measured urinary excretion of catecholamine and reported no significant change in total catechol excretion among cardiac patients. Later, he ${ }^{2)}$ succeeded in measuring catecholamine in the myocardium, demonstrating increase of neurohormones in myocardial infarction and congestive heart failure, especially in acute cardiac failure or pulmonary edema. He also stated ${ }^{3)}$ that the characteristics of metabolism of the failing heart closely resembled that elicited by administration of catecholamine. Pekkarine ${ }^{4)}$ stated that the urinary level of excretion of catecholamine was increased in the moderately severe cases of congestive heart failure and there was increased

From the Department of Internal Medicine, Division I, Kobe Medical College, Kobe. 
excretion of noradrenaline in small number of the patients studied. Bloomworth and E. von Haam, ${ }^{5)}$ however, could demonstrate no significant increase of tissue catecholamine in any cardiacs. Thus, there are no consensus of opinion in this respect.

Since reduction or disappearance of the humoral principle, chiefly catecholamine would be responsible for the development of the failing heart in the heart-lung preparation, as pointed by Kako et al., (6) catecholamine in congestive heart failure should be a worthy problem for the investigation.

Authors have made an attempt in this paper to deal with measuring urinary excretion of catechol in congestive heart failure and try to interpret its significance.

\section{Material and Method}

The patients who were suffering from the congestive heart failure caused by acquired valvular disease were chosen for the measurement of catechols in 24-hour urine.

Among those who were admitted to the ward of Kobe Medical College, 178 patients with congestive heart failure caused by acquired valvular disease were chosen, excluding those with hypertensive, sclerotic, or coronary heart and congenital heart diseases. The patients were divided into 4 groups according to the criteria of New York Heart Association. There were 5 patients in grade I, 81 in grade II, 67 in grade III and 25 in grade IV.

Adrenaline, noradrenaline, acid catechols and 3,4-dihydroxyphenylethylamine (Dopamine) were determined on 24-hour urine. The method employed for catechols mesurement was already reported in the other paper. Catecholamine in urine adsorbed on alumina was eluted with $0.4 \mathrm{~N}$ acetic acid solution. The elute thus obtained was divided into active catechols, Dopamine and acid catechols by means of column chromatography with Duolite $\mathrm{C}_{25}$ resin. THI method was employed for determining active catechols, and ED method for Dopamine and acid catechols.

\section{Results}

Adrenaline, noradrenaline, acid catechols and Dopamine measured in 178 cardiacs are indicated in Table I and Fig. 1-4. Comparing with the average values obtained from 30 normal subjects both adrenaline and noradrenaline measured were found to be increased in average. In patients in grade III a distinct rise in urinary excretion of these 2 hormones was noted, on the other hand, those in grade IV showed a lower excretion, though still higher than the normal average (Fig. 1, 2). Ratio of adrenaline to noradrenaline is approximately $1: 4$ in urine of 
Table I. Urinary Level of Excretion of Catechols in Normal Subjects and Patients with Cardiac Failure Duc to Valvular Diseases

\begin{tabular}{|c|c|c|c|c|}
\hline $\begin{array}{r}\text { Catechols } \\
\gamma / \text { day }\end{array}$ & Adrenaline & Noradrenaline & Acid Catechols & Dopamine \\
\hline $\begin{array}{c}\text { Normal Subjects } \\
(30 \text { cases })\end{array}$ & $\begin{array}{l}5.2 \pm 1.7 \\
(0.21)\end{array}$ & $\begin{array}{c}20.1 \pm 4.8 \\
(0.79)\end{array}$ & $245 \pm 46.0$ & $34.1 \pm 4.8$ \\
\hline $\begin{array}{c}\text { Cardiac Failure } \\
\text { Grade I } \\
\text { ( } 5 \text { cases })\end{array}$ & $\begin{array}{l}4.2 \\
(0.18)\end{array}$ & $\begin{array}{l}19.6 \\
\quad(0.82)\end{array}$ & 274 & 43.0 \\
\hline $\begin{array}{c}\text { Cardiac Failure } \\
\text { Grade II } \\
\text { (81 cases) }\end{array}$ & $\begin{array}{c}9.2 \pm 1.7 \\
(0.20)\end{array}$ & $\begin{array}{c}36.7 \pm 10.3 \\
(0.80)\end{array}$ & $298 \pm 111.5$ & $46.1 \pm 15.5$ \\
\hline $\begin{array}{c}\text { Cardiac Failure } \\
\text { Grade III } \\
(67 \text { cases })\end{array}$ & $\begin{array}{l}19.7 \pm 4.4 \\
(0.24)\end{array}$ & $\begin{array}{l}62.2 \pm 15.6 \\
\quad(0.76)\end{array}$ & $604 \pm 140.6$ & $64.8 \pm 16.3$ \\
\hline $\begin{array}{c}\text { Cardiac Failure } \\
\text { Grade IV } \\
(25 \text { cases })\end{array}$ & $\begin{array}{c}7.5 \pm 1.0 \\
(0.17)\end{array}$ & $\begin{array}{c}37.3 \pm 9.4 \\
(0.83)\end{array}$ & $284 \pm 90.3$ & $44.1 \pm 15.5$ \\
\hline
\end{tabular}

Note: Figures in parentheses mean $\frac{\text { Adrenaline or Noradrenaline }}{\text { Adrenaline+Noradrenaline }}$

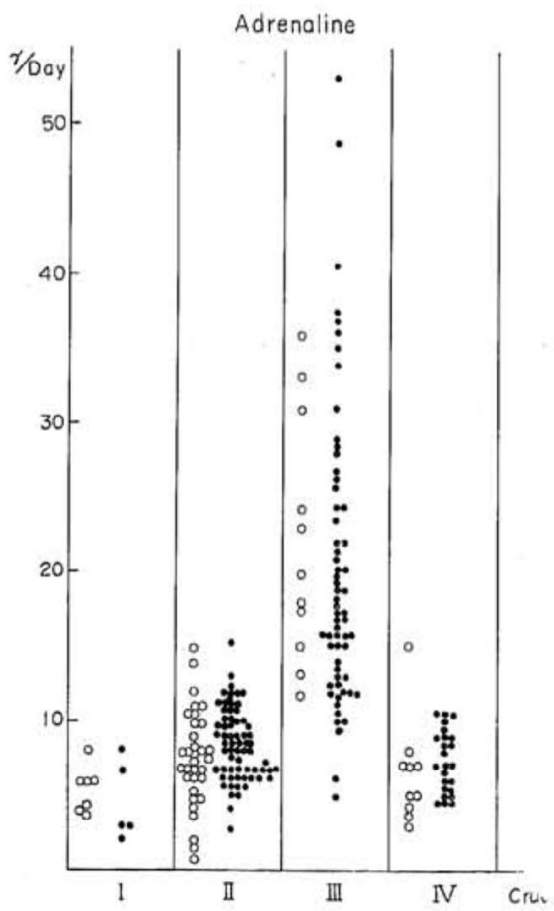

Fig. 1. Urinary excretion of adrenaline of patients with cardiac failure due to valvular and hypertensive cardiac diseases.

- valvular disease

$O$ hypertensive cardiac disease 


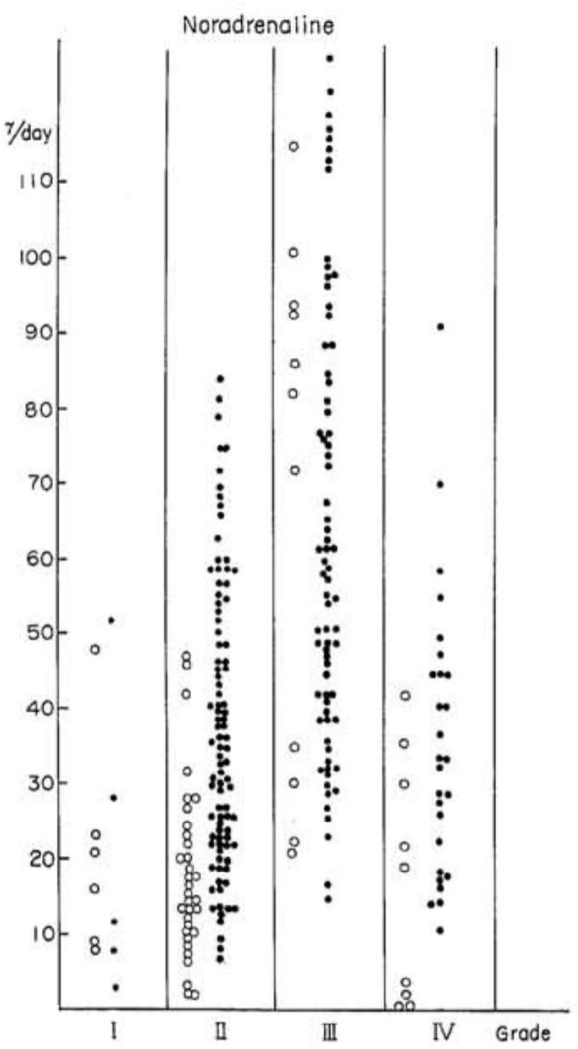

Fig. 2. Urinary excretion of noradrenaline of patients with cardiac failure due to valvular and hypertensive cardiac diseases.

normal subjects, in other words, the amount of adrenaline occupies about 20 per cent in the total urinary excretion of active catecholamine. In patients in grade I, II and IV urinary excretion of adrenaline took almost identical percentage in total active catecholamine but those in grade III gave a little higher ratio of adrenaline (Table I). As shown in Fig. 2, values of noradrenaline scattered in fairly wide range were presumably attributed to the patients' condition on the day of collecting urine. On the contrary, values of adrenaline were rather fixed except in patients in grade III.

So far as acid catechol and Dopamine are concerned, there was no correlation of urinary excretion of these substances to the degree of congestive heart failure. 


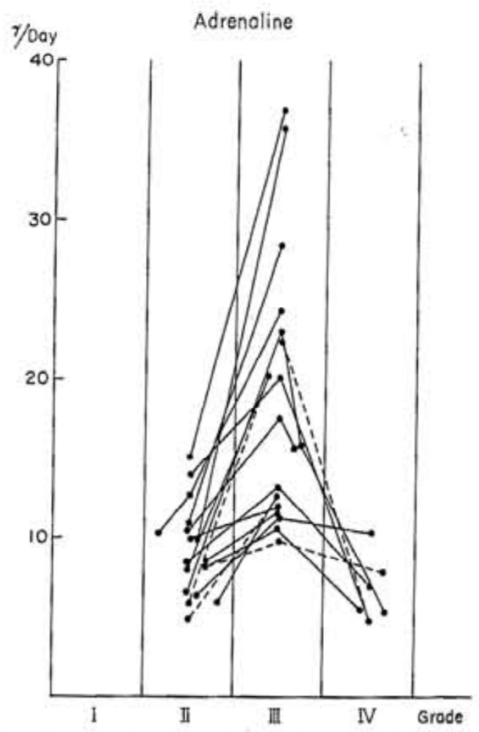

Fig. 3. Variation of urinary level of adrenaline in patients with cardiac failure during the course of treatment.

The straight line $(-)$ indicates the direction of changeable values from grade IV to III or grade III to II; the dotted line (-..) indicates the opposite direction to the former, namely from grade III to IV or grade II to III.

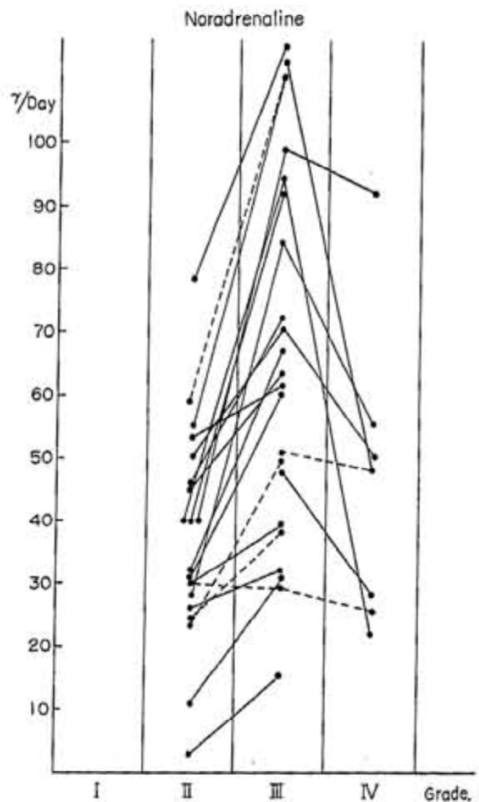

Fig. 4. Variation of urinary level of noradrenaline in patients with cardiac failure during the course of treatment.

The straight and dotted lines indicate the same as in Fig. 3. 
It has been demonstrated by measurement made along with the clinical course of some patients that urinary excretion of both adrenaline and noradrenaline had increased when the condition was improved from grade IV to grade III and reduced with further restoration from grade III to grade II. When the condition took a turn for the worse, namely from grade II to grade III or from grade III to grade IV, urinary excretion of active catecholamine varied inversely (Fig. 3 and 4)

Fig. 5 shows one of the examples presenting diurnal change of

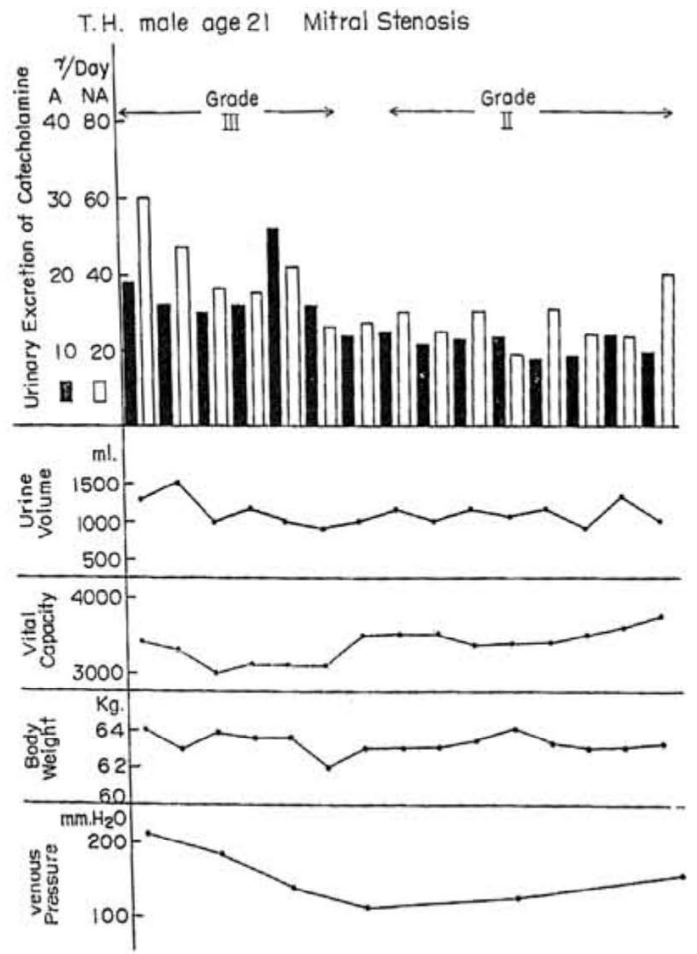

Fig. 5. Diurnal change of urinary excretion of active catechols and some clinical features in a 21 -year-old male patient with mitral stenosis.

urinary active catechol in a 21 -year-old male with mitral stenosis under treatment. The patient's conditions gradually improved during the observation period of 7 weeks as seen in some clinical manifestations, such as urinary volume, body weight, vital capacity and venous pressure. Urinary excretion of catecholamine was gradually decreased with improvement of the patient's symptoms from grade III to grade II. To be exact, urinary adrenaline was between 15 and $25 \mathrm{r} /$ day in the first week of observation and urinary noradrenaline was between 35 and $60 \mathrm{r} /$ day. 
During the ensuing few weeks urinary excretion of both hormones was 10-15 r/day and 20-30 r/day respectively. Ratio of adrenaline to total active catechol was constantly over 20 per cent. The measurements of urinary active catechol were made on every 5- or 6- hour urine during the course of the rapid digitalization and compared with some clinical manifestations including urinary volume, vital capacity, body weight and venous pressure.

In a 60-year-old male patient with mitral stenosis and insufficiency, grade IV, the rapid digitalization was tried with Digilanogen $\mathrm{C}$ (Lanatoside C), $0.4 \mathrm{mg}$. every 4 hours, 3 times on the first day and once on the following day (Fig. 6). With abating the congestive state, urinary excretion of

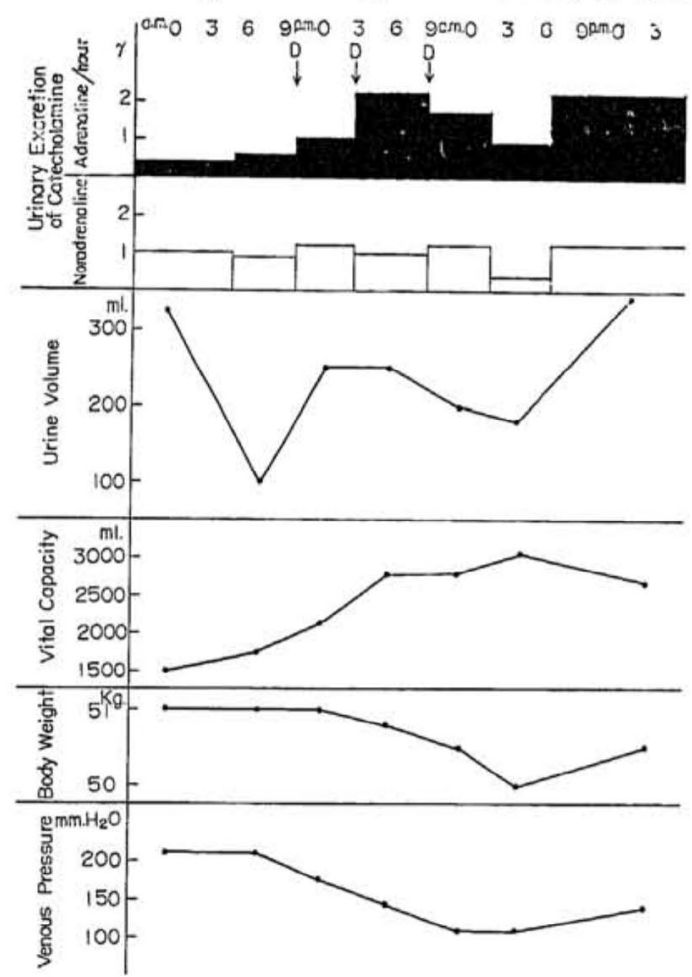

Fig. 6. Y.Y., 60-year-old male, with mitral stenosis and insufficiency, grade IV.

Successive observation of variations of urinary level of active catechols in short-term period (every 5- to 6-hour urine) before and during rapid digitalization.

adrenaline grew progressively along with the increasing dose of digitalis and reached to the maximum at the approximately saturated state with the drug in spite of a little change in noradrenaline. Fig. 7 shows the variation of catecholamine in series of 6-hour urine during digitalization 


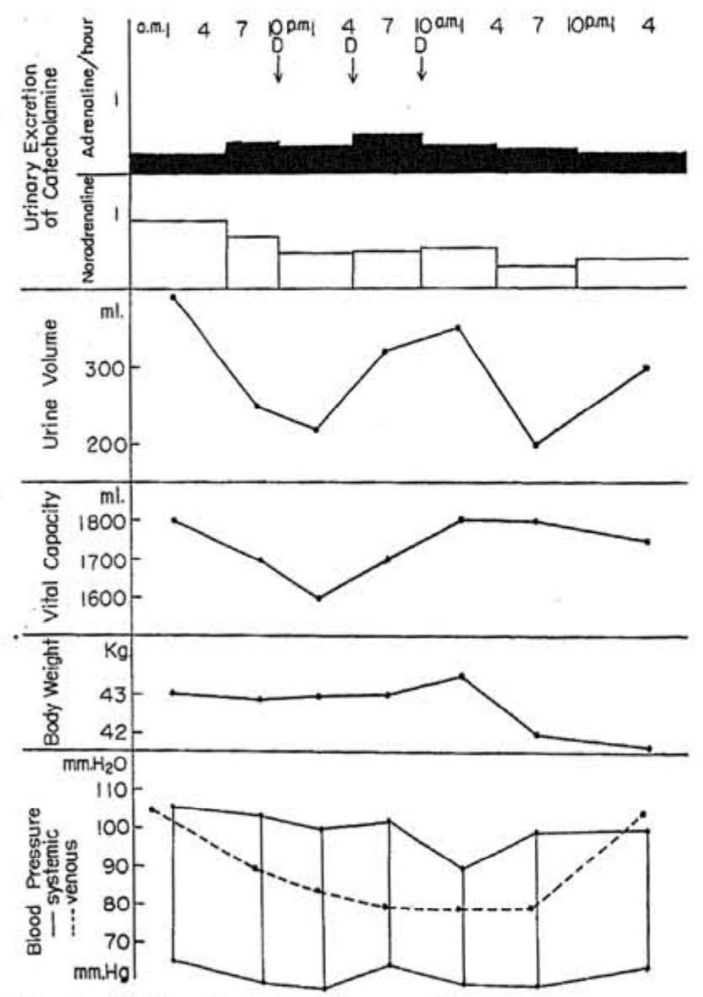

Fig. 7. C.N., female, 34-year-old, mital stenosis, grade I. Successive observation of variation of urinary level of active catechols in short-term period (every 5- to 6-hour urine) before and during rapid digitalization.

in a 34-year-old female patient with mitral stenosis, grade II, rather milder case, with constant ambiguous complains due to nervousness. Treatment with digitalis brought about less significant improvement of clinical symptoms and signs and resulted in little change in urinary excretion of catecholamines.

Fig. 8 shows a 41 -year-old male with mitral stenosis and insufficiency associated with atrial fibrillation who was admitted into our ward due to the critical state with orthopnea, generalized edema and ascites after repetitive decompensations since 1960 . Digilanogen C $0.4 \mathrm{mg}$. was given intravenously every 4 hours. Urinary excretion of adrenaline and noradrenaline was extremely low before digitalization, but increased exceedingly in the first 4-hour urine. Then, urinary excretion of noradrenaline was decreased but still far above normal range; urinary excretion of adrenaline was decreased very slightly. After all, urinary excretion of catecholamine was increased markedly. At the same time orthopnea had subsided with increase in vital capacity; increase in urine volume and 


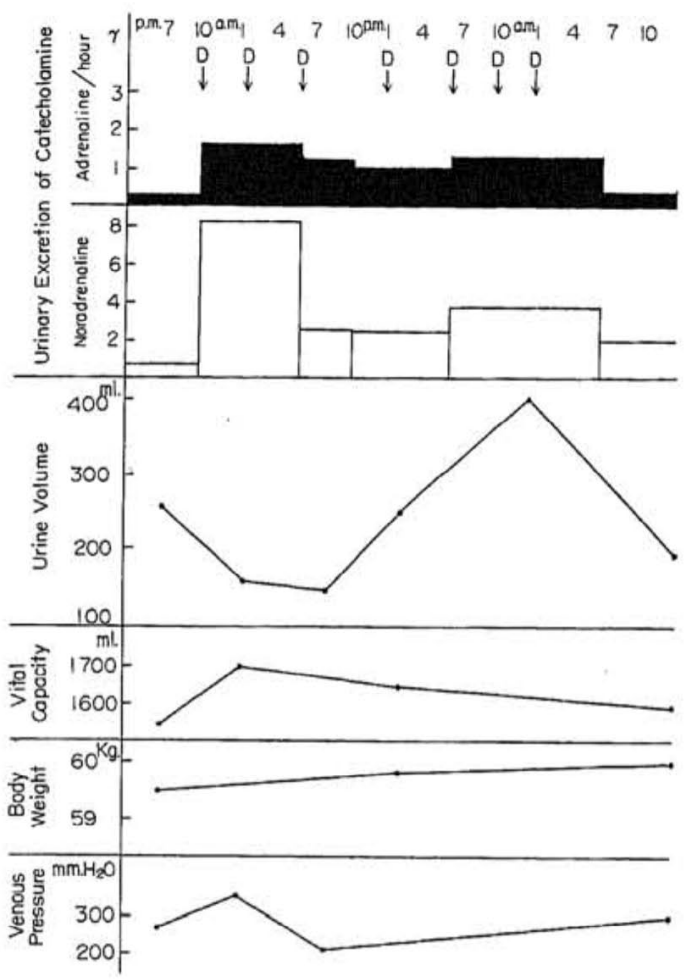

Fig. 8. A.N., male, 41-year-old, mitral stenosis and insufficiency, grade IV.

Successive observation of variations of urinary level of active catechols in short-term period (every 5- to 6- hour urine) before and during rapid digitalization.

lowering of venous pressure followed; yet inotropic effect of digitalis was found not to be sufficient. As a result, improvement was limited and tentative, but further increase in urinary excretion of catecholamine was not seen. The patient died suddenly 5 days after the beginning of digitalis treatment. The last measurement gave $8.1 \% /$ day of adrenaline and $4.5 \gamma /$ day of noradrenaline.

\section{Discussion}

Urinary excretion of catecholamine was found to be increased in congestive heart failure due to acquired valvular disease. Increment of each active catechol was proportional in average but value of noradrenaline were scattered in wide range. It might be attributed to the emotional state under which the patients were put when examined.

The question as to whether increased urinary excretion of nor- 
adrenaline is one of the signs properly complicated by congestive heart failure or just one of the factors responsible for modifying the clinical manifestation of the cardiacs requires further investigation. In fact, several week observations of diurnal change of urinary catecholamine, though confined to the small number of cases, indicated comparatively stable value while the patients were maintained in rest. Values of adrenaline were fairly fixed except in patients in grade III.

It is interesting that both hormones were found to be the highest in patients in grade III and decreased in those in grade IV, though even above normal range. This is also proved by the fact that urinary catecholamine is increased when the clinical conditions of the cardiacs took a turn from grade II to grade III and decreased when from grade III to grade IV, and that urinary catecholamine varied conversely when the patient's conditions took a turn for the worse. The early report of $\mathrm{Raab}^{1)}$ showed no significant increase in urinary excretion of catecholamine in cardiacs, but Pekkarine ${ }^{4)}$ stated that some increase in urinary catechol was found in congestive heart failure of moderate severity. The latter's data is fundamentally in accord with ours, however, he failed to mention an enormous increase as we have seen.

It is still under controversy whether urinary level of catecholamine can be an indicator for the circulating catecholamine. Raab ${ }^{8}$ stated that exogenous catecholamine was selectively absorbed by the heart muscle and other vascular tissues, but von Euler ${ }^{9)}$ was opposed to it, observing no definite increase of catecholamine in the heart after injection of either small or large dose of catecholamine.

Using $\mathrm{H}^{3}$ labelled catecholamine Axelrod et al. ${ }^{10)}$ proved rapid and selective uptake and retention of catecholamine by the heart. Therefore, urinary excretion of catecholamine may be modified by uptake of circulating catecholamine by the cardiovascular system. When digitalis failed to improve cardiac insufficiency, urinary excretion of catecholamine remained unchanged; when digitalis was used successfully urinary catecholamine, especially adrenaline was elevated. With incomplete digitalis effect even though there was a presence of transitory effect, urinary catecholamine was found to be a transient increase though it was eventually lowered. Thus, it would suggest the possibility of uptake as well as retention of catecholamine by the heart before administration of digitalis. It is assumed that catecholamine retained might contribute to maintenance and augmentation of contractility of the myocardium, though in low level of efficiency. Both that urinary excretion of adrenaline increased more than that of noradrenaline, during the treatment with digitalis, and that observation of diurnal change in the urinary level of catecholamine seen in Fig. 7 showed more urinary excretion of adrenaline 
than that of noradrenaline, while the patient was kept in rest, are not always in accord with the results that urinary level of noradrenaline covered the wide range and ratio of adrenaline to the total catecholamine was limited to nearly normal range except in patients in grade III in which ratio of adrenaline showed a little higher value. Under such circumstances more additional results should be evaluated before drawing any final conclusion.

Considering (1) Sarnoff's ${ }^{11)}$ statement that ventricular contraction can be augmented with catecholamine and (2) Raab's document that a marked increase of catecholamine was found in the myocardium of myocardial infarction, congestive failure and acute pulmonary edema, inference could be drawn that increase in circulating catecholamine and increased uptake by the myocardium were found in congestive heart failure, contributing to augmentation of myocardial contraction. Since it has been demonstrated, (1) that catecholamine displays inotropic action on the heart at the expense of excessive consumption of oxygen, ${ }^{12)}$ (2) that a marked elevation in serum transaminase including serum glutamic oxalacetic and glutamic pyruvic transaminase, lactic dehydrogenase and alkaline phosphatase were observed after an injection of large doses of catecholamine just as seen after coronary occlusion, ${ }^{13)}$ and (3) that histological changes of myocardium such as myofibril degeneration or necrosis was produced by infusion of $l$-norepinephrine, ${ }^{14)}$ the myocardium would be deteriorated by an increased catecholamine, especially norepinephrine. Therefore, it should be assumed that catecholamine could exert the beneficial effect on myocardial contraction under a certain limitation. It seems to be still far from drawing any conclusion on the question as to whether adrenaline or noradrenaline can exercise the favorable effect on myocardial contraction.

\section{Conclusion}

Determining urinary excretion of catechols in 178 patients with congestive heart failure caused by acquired valvular disease, tentative conclusion can be drawn as follows:

(1) Urinary excretion of catechols, especially adrenaline and noradrenaline, was found to be increased.

(2) Values of both urinary adrenaline and noradrenaline were progressively increased from grade I to grade III of congestive heart failure and decreased in grade IV but not to the normal range.

(3) Values of urinary noradrenaline covered a wide range.

(4) Some comments were extended on the significance of urinary excretion of active catechol. 


\section{REFERENCES}

1. Raab, W. and Gigee, A.B.: Circulation 9: 592, 1954.

2. Raab, W. and Gigee, A.B.: Circulation 11: 593, 1955.

3. Raab, W.: Am. J. Cardiol. 5: 571, 1960.

4. Pekkarine, A., Iisalo, E., Kasanen, A., Leihinen, A., and Thomason, B.: Am. J. Cardiol. 5: 604, 1960.

5. Bloomworth, J.M.B. and von Haam, E.: Circulation 13: 573, 1956.

6. Kako, K., Chrysohon, A., and Bing, R.J.: Am. J. Cardiol. 6: 1109, 1960.

7. Ueba, Y., Matsumoto, T., and Ikoma, T.: to be published.

8. Raab, W. and Gifee, A.B.: Circulat. Res. 3: 553, 1955.

9. von Euler, U.S.: Circulat. Res. 4: 647, 1956.

10. Axelrod, J., Whitley, G., Hertting, G., and Kopin, I.J.: Circulat. Res. 9: 715, 1961.

11. Sarnoff, S. J.: Am. J. Cardiol. 5: 579, 1960.

12. Gollwitzer-Meier, Kl.: Verh. d. Dtsch. Ges. Kreislaufforsh. 16. Tagung 3, 1950.

13. Maling, H.M., Highman, B., and Thompson, E.C.: Am. J. Cardiol. 5: 628, 1960.

14. Szakacs, J.E. and Mehlman, B.: Am. J. Cardiol. 5: 619, 1960. 